\title{
Role of tumor suppressor p53 and micro-RNA interplay in multiple myeloma pathogenesis
}

\author{
Jahangir Abdi ${ }^{1,2}$, Nasrin Rastgoo ${ }^{1,2}$, Lihong $\mathrm{Li}^{3}$, Wenming Chen ${ }^{3}$ and Hong Chang ${ }^{1,2,4^{*}}$
}

\begin{abstract}
The molecular mechanisms underlying dysregulated wild type (wt) p53 in multiple myeloma (MM) have been subjects of intense investigation for years. Indeed, correlation of rarely occurring TP53 gene mutations or deletions with adverse clinical outcomes in MM patients is strongly established, while in majority of cases wtp53 seems to be non-functional or dysregulated bearing a high clinical impact. Interestingly, findings from recent investigations show that micro-RNAs (miRNAs) may contribute to suppression of wtp53 in MM, as they are now known to function as key regulatory elements in the p53 network. This area is shedding new light on understanding the biologic effects of dysregulated p53 in MM pathogenesis especially drug resistance. miRNAs such as miR-125b (oncomiR) or miR-34a (tumor suppressor-miR) can be negative or positive regulators of wtp53 function, respectively, with specific effects on MM cell viability. On the other hand, our knowledge of miRNA interaction with mutant (mt) p53 in MM, which is rather related to disease progression and resistance to therapy, is limited which demands in-depth exploration. Here, we will put forward the current knowledge on miRNA-p53 interaction in MM and its role in MM pathogenesis including drug resistance. We will also highlight the pre-clinical approaches for therapeutic application of miRNAs targeting p53 pathway.
\end{abstract}

Keywords: p53, Micro-RNA, Myeloma

\section{Background}

In multiple myeloma (MM), p53 is frequently dysregulated culminating in abnormal function of p53 signaling pathway and its related downstream targets [1], while mutations or deletions in TP53 gene are rare phenomena in MM, with approximate rates of 3 or $10 \%$, respectively, at diagnosis as reported by us and others [2-5]. These observations reflect the fact that inactivation of a wild-type functional p53 (wtp53) could be the major contributor to dysregulation of p53 pathway, hence MM pathogenesis especially drug resistance. Accordingly, extensive research has been focusing on deciphering the mechanisms of dysregulation of p53 pathway and related targets. As reported by us [6-8] and other groups [9, $10]$, non-genotoxic targeting of the pathway using small

\footnotetext{
* Correspondence: hong.chang@uhn.ca

'Division of Molecular and Cellular Biology, Toronto General Research Institute, Toronto, Canada

${ }^{2}$ Department of Laboratory Medicine \& Pathobiology, University of Toronto, Toronto, Canada

Full list of author information is available at the end of the article
}

molecules indicates that negative regulators of p53 including MDM2 contribute largely to p53 pathway dysregulation in MM. However, elucidation of the mechanisms underlying p53 pathway dysregulation in MM demands more detailed studies.

Micro-RNAs (miRNAs) are small non-coding RNAs $(\sim 20-24 \mathrm{nt}$ in length) that are involved in the posttranscriptional control of gene expression. miRNAs generally bind to target mRNAs through their $3^{\prime}$ untranslated regions (UTRs) and recruit the RNA-induced silencing complex (RISC), which mediates the inhibition of translation and the degradation of the respective mRNA [11]. It is estimated that $>60 \%$ of human protein coding genes may be subject to regulation by miRNAs [12]. Among these targets, the tumor suppressor gene TP53 is of particular importance as it can regulate expression and processing of miRNAs and the mRNA of TP53 can be a direct target of miRNAs [13]. Interestingly, it has been suggested that miRNAs may have evolved to provide the cells with the ability to effectively deal with 
stress $[14,15]$. This concept further supports the notion that p53 stress response pathway would have a strong interrelationship with miRNAs. Moreover, a great body of evidence has now established miRNAs as critical players in MM pathogenesis, progression, and therapy resistance [16-19].

In recent years, some studies in MM have taken advantage of the role of miRNAs in attempts to better understand the mechanisms underlying p53 dysregulation and its contribution to drug resistance, the critical aspect of MM pathogenesis. However, these studies focused mainly on interaction of wtp53 with miRNAs in MM; thus, whether/how miRNAs interact with mutant p53 (mtp53) to possibly explain the resistance to therapy or relapse in MM patients carrying $\mathrm{mt} 53$ is still not clear. This review will discuss the current understanding of p53-miRNA interaction and its role in MM pathogenesis especially drug resistance. Potential therapeutic applications of miRNAs which targetp53 pathway will also be highlighted.

\section{miRNAs in a p53 network: control of MM cell viability and drug response}

The tumor suppressor p53 is inactivated in almost all human cancers while only 50\% harbor TP53 mutations, particularly in MM where this rate may be as low as $3 \%$ early at diagnosis. Moreover, mono allelic deletion of TP53 is detected only in approximately 10\% of MM patients at diagnosis [2-5]. These observations highlight the fact that suppressed p53 or frequent dysregulation of p53 signaling pathway in MM emerges even in the face of a functional wtp53. Interestingly, as demonstrated by several studies in recent years in MM $[20,21]$ and other cancers [22], miRNAs may have an essential role in lowering p53 expression or activity. Hundreds of genes and their products establish a complex network comprising p53, its regulators, and regulated genes which collectively help p53 maintain its proper function [1]. Recent studies have demonstrated that miRNAs interact with p53 and its network at multiple levels. Thus, p53 can regulate the transcription expression and the maturation of a group of miRNAs, while miRNAs can regulate the activity and function of p53 through direct repression of p53 or its regulators. These findings indicate that miRNAs are important components in the p53 network and add another level of complexity to the p53 network [23].

Understanding the mechanisms behind dysregulation of functional p53/p53 signaling pathway in MM will shed new light on pathogenesis particularly drug resistance and refractoriness of the disease. Due to their versatility in gene regulation, miRNAs have recently been identified as factors capable of modulating viability and drug response of MM cells in relation to their p53 status. The mutual functional link between miRNAs and p53 in cancers including MM has provided some important clues to the mechanisms underlying p53/p53 pathway dysregulation which will in turn open new venues to MM therapy. In two separate sections as follows, we will discuss the biologic outcomes of such interaction for two categories of miRNAs, negative and positive regulators of $\mathrm{p} 53$ function.

\section{iRNAs as positive regulators of wtp53 in MM cells: $a$ suppressed positive feedback loop threatens viability}

It is well established that p53 as a transcription factor can directly regulate the expression of a growing number of miRNAs (Table 1). This indicates that p53 can transactivate tumor suppressor (TS)-miRs or repress some of the oncomiRs. miRNAs transactivated by p53 mainly target the anti-apoptotic and anti-proliferative genes, hence boosting the tumor suppressor activity of p53, or they can even regulate p53 itself in a positive feedback loop. On the contrary, miRNAs repressed by p53 may target the tumor suppressor or pro-apoptotic genes leading to dampening tumor suppressor activity of p53 [24] (concept illustrated in Fig. 1).

miRNAs which activate p53 act indirectly by interacting with p53-regulating factors. These miRNAs include miR-122, 34, 29, 192, 194, 215, and 605 which activate (regulate abundance/activity of) p53 by binding to the 3' UTR of factors like MDM2, SIRT1, and YY1 [13]. The first indication of a miRNA transcriptionally regulated by wtp53 was reported when several studies found miR34 family members miR-34a/b/c to be directly regulated by wtp53 [25, 26]. miR-34 family members are wellknown TS-miRs which repress the expression of several targets involved in regulation of cell cycle and proliferation, like BCL-2, Notch1, cyclin E2, cyclin-dependent kinases 4 and 6 (CDK4 and CDK6), in MM [27] and other cancers [26]. Moreover, miR-34a has been identified to be hypermethylated at its promoter region in MM [28]. Notably, in one study, the expression of miR-34a was correlated with TP53 mutational status and del17p.13 (by fluorescent in situ hybridization (FISH)) in human myeloma cell lines (HMCLs) [29], consistent with the notion that miR-34a is transactivated by wtp53 [30]. Mutations in exons 5 and 8 of TP53 were identified in all HMCLs with low miR-34a. In particular, del17p.13 by FISH was noted in KMS11 cells $\left(T P 53^{N U L L}\right)$, consistent with their very low or undetectable miR-34a. Furthermore, overexpression of miR-34a in KMS11 and OPM2 cell lines reduced the level of $\mathrm{Bcl}-2$, CDK4, CDK6, CEBP $\alpha$, and YY1, sensitized them to bortezomib (BTZ) and reduced tumor growth in vivo. Anti-myeloma activity of miR-34a was reported in another study showing the killing effects of miR34a mimics in xenograft harboring TP53-mutated MM cells [27]. Additionally, low miR-34a level was reported to be correlated with low p53 expression and resistance to fludarabine in CLL [31], further supporting the role of miR-34a in regulation of p53-mediated tumor cell death. 


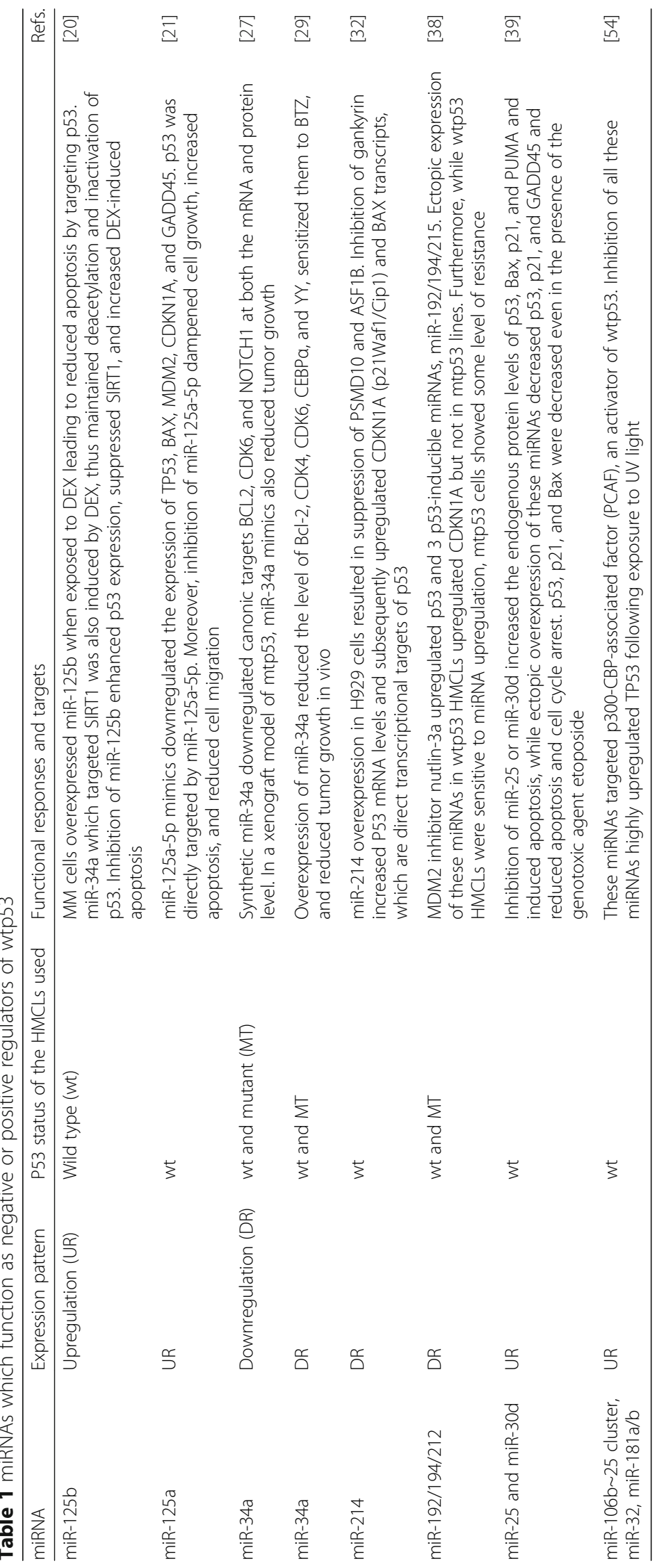




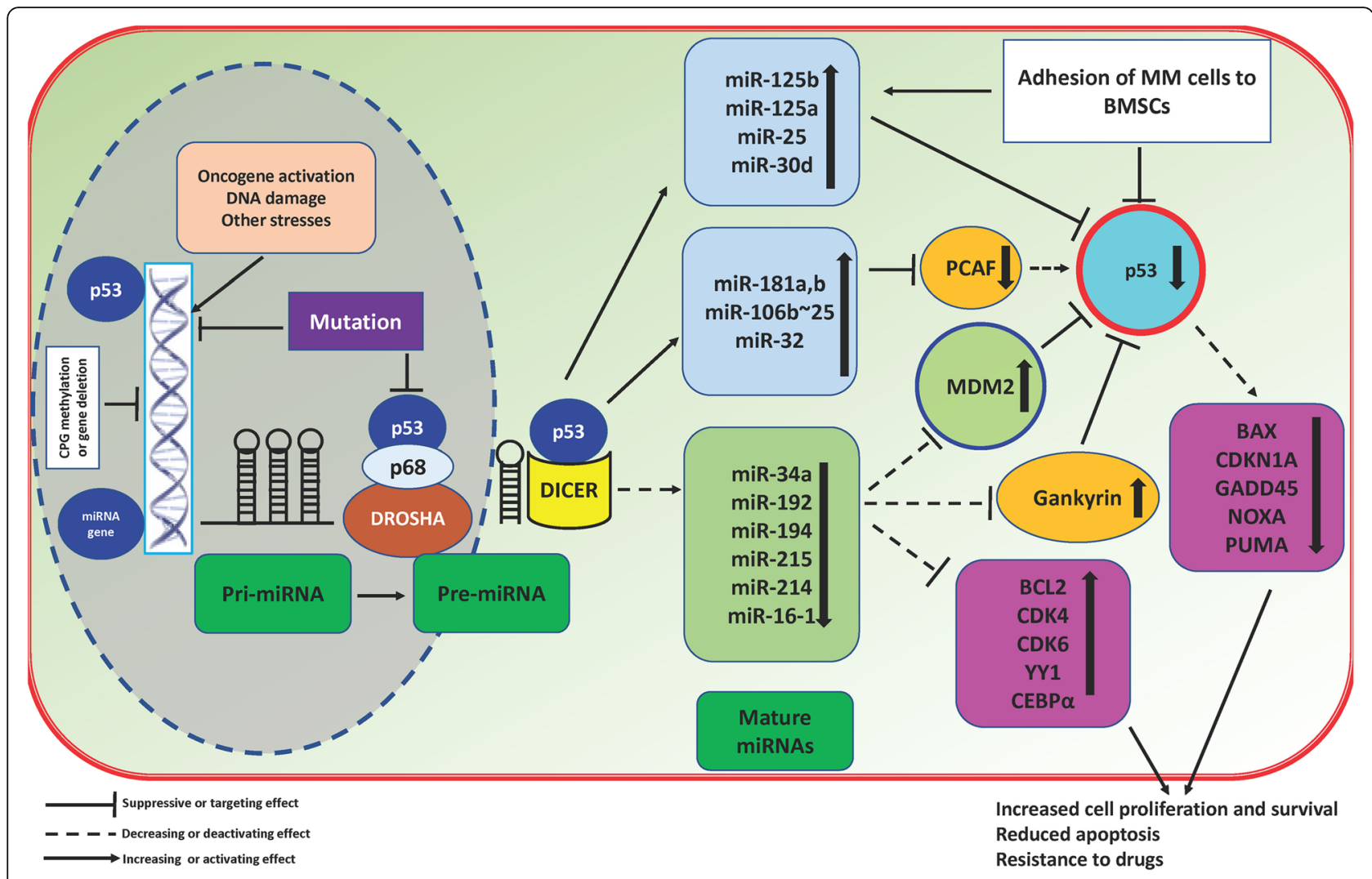

Fig. 1 Schematic mechanism of p53-miRNA interaction in MM. TS-miRs known to be regulated by wtp53 (e.g., miR-192) target negative regulators of wtp53 like MDM2 and gankyrin or target cell cycle-related/anti-apoptotic proteins to enhance tumor suppressor activity of wtp53. However, this scenario is compromised in MM context possibly due to epigenetic silencing of TS-miRs or wtp53 and also due to upregulation of negative regulators. Therefore, the outcome will be a suppressed wtp53 which ends in downregulation of p53 downstream targets such as PUMA, BAX, and NOXA, maintenance of MM cells survival, and probably resistance to anti-myeloma drugs. On the other hand, oncomiRs (e.g., miR-125b) negatively regulate p53 either by directly targeting p53 or by targeting positive regulators of p53 (such as PCAF) which culminates in the same outcomes as above. Of note, wtp53 can also regulate miRNA biogenesis by association with DROSHA or DICER, a function which is completely antagonized by mtp53. In MM, mtp53 may function as an oncogene by interfering with biogenesis of TS-miRs which could partly explain the resistance of mtp53 phenotypes to anti-myeloma drugs (however, this concept demands in-depth investigation in MM)

miR-214 is another miRNA which has been reported to positively regulate p53 abundance and function in wtp53 HMCLs [32]. Ectopic expression of miR-214 induced apoptosis and reduced cell growth in wtp53carrying H929 cells and suppressed PSMD10 (which encodes the oncoprotein gankyrin, a p53 negative regulator) and ASF1B (a histone chaperone required for DNA replication), which were found to be direct targets of miR-214. Inhibition of gankyrin increased P53 mRNA levels and subsequently upregulated CDKN1A (p21Waf1/ Cip1) and BAX transcripts, which are direct transcriptional targets of $\mathrm{p} 53$. These results indicate that miR-214 functions as a tumor suppressor in MM by positive regulation of p53 and inhibition of DNA replication. Of note, high level of miR-214 in the serum of MM patients was reported to be correlated with aggravated bone disease and poor outcome [33]. However, this study did not provide any evidence that miR-214 displays the same expression and function (as an oncomiR) in the tumor cells from the bone marrow or in the HMCLs. Further, they could not find any correlation of high serum miR-214 with MM patients' response to BTZ and argued that high miR-214 in the serum was much more associated with myeloma bone destruction rather than tumor burden. Additionally, miR-214 regulated cancer stemness in solid tumors by targeting p53 and Nanog [34], suggesting context-dependent expression and function of miR-214.

A cluster comprising miR-192, miR-194, and miR-215 has been identified as p53-induced miRNAs in several cancer types including MM [35-38]. Pichiorri et al. [38] provided evidence that miR-192, miR-194, and miR-215 are transcriptionally induced by p 53 and they, in turn, activate p53 indirectly by targeting MDM2 leading to p21 upregulation and induced apoptosis and cell cycle arrest in wtp53 HMCLs. Introduction of these miRNAs into wtp53 HMCLs (MM.1S, H929, KMS28BM) led to upregulation of CDKN1A and downregulation of MDM2 in mtp53 cell lines but no change at TP53 mRNA. 
Interestingly, miRNA transfection in wtp53 cells with higher MDM2 level (MM.1S and H929) induced G0/G1 arrest, while in wtp53 cells with lower MDM2 level (KMS28BM), it triggered sub-G0 accumulation (apoptotic cell death). These findings clearly indicate that the p53-induced miR-192, miR-194, and miR-215 control MM cell viability by targeting MDM2.

\section{miRNAs as negative regulators of wtp53 function in MM cells: a negative feedback loop threatens viability}

Several miRNAs have been described to negatively regulate p53 by directly binding to its 3'UTR: miR-25 and miR-30d [39], miR-125b, [40] miR-504, [41] miR-380-5p, [42] miR-92, and miR-141 [43]. However, only a few of them have been functionally explored in relation to p53 in MM (Table 1). Kumar et al. [39] observed that miR25 and miR-30d were overexpressed around 6.3- and 11.2-fold, respectively, in plasma cells from MM patients compared with those from healthy donors. Inhibition of miR-25 or miR-30d increased the endogenous protein levels of p53, Bax, p21, and PUMA and induced apoptosis, while ectopic overexpression of these miRNAs decreased p53, p21, and GADD45 and reduced apoptosis and cell cycle arrest. The authors further observed that p53, p21, and Bax were decreased even in the presence of the genotoxic agent etoposide implying a suppressive role for $\mathrm{miR}-25 / \mathrm{miR}-30 \mathrm{~d}$ in $\mathrm{p} 53$-mediated apoptosis.

mir-125b is the most important p53-targeting oncomiR to be studied in MM pathogenesis especially drug resistance because (a) it has been shown to play an eminent role in normal lymphopoiesis or myelopoiesis and also to function as an oncomiR in hematologic malignancies at least by targeting p53 [44], (b) latter function of miR-125b is further supported by the observations that it also targets other components of the p53 pro-apoptotic network including BAK1, PUMA, BMF, TRP53INP1, and Kruppellike factor 13 (KLF13) [45, 46], (c) miR-125b proved to be a leukemogenic oncomiR in mouse models by causing acute myelogenous leukemia (AML) and acute lymphoblastic leukemia (ALL) phenotypes [47, 48], and (d) miR125b was reported to be associated with drug resistance in ALL [49, 50]. Megan et al. described a mechanism whereby MM cells overexpressed the oncogenic miR-125b when exposed to dexamethasone (DEX) leading to reduced apoptosis, and they further showed direct targeting of TP53 mRNA by miR-125b [20]. Interestingly, miR-34a which targeted SIRT1 deacetylase was also induced by DEX, hence allowing maintenance of deacetylation and inactivation of p53. Strikingly, inhibition of miR-125b enhanced expression of p53, repressed expression of antiapoptotic SIRT1, and, importantly, significantly enhanced DEX-induced cell death in MM cells. These findings suggest that resistance against chemotherapy in MM cells works greatly via the p53/miR-34a/SIRT1 signaling network which provides a platform for therapeutic intervention.

miR-125a, which shares the same seed sequence with miR-125b [51], has also been reported to show the same expression pattern and function as miR-125b in hematologic malignancies including diffuse large B cell lymphoma (DLBCL) [52]. In MM, Leotta et al. [21] showed that when MM cells were transfected with miR125a-5p mimics, expression of TP53 gene and p53 pathway-related genes such as BAX, MDM2, CDKN1A, and GADD45 was downregulated, and p53 was found to be a direct target of miR-125a-5p. Moreover, inhibition of miR-125a-5p expression in wtp53 MM cells dampened cell growth, increased apoptosis, and reduced cell migration. These findings strongly support the oncogenic function of miR-125a-5p in MM cells. Notably, miR-125a was associated with resistance to daunorubicin in leukemia cell lines further suggesting its role in drug resistance as an oncomiR [53].

It has also been shown that in MM cells, wtp53 can be indirectly targeted by miRNAs. Pichiorri et al. found that miR-106b 25 cluster, miR-32, and miR-181a/b were overexpressed in MM cells relative to normal controls [54]. These miRNAs targeted p300-CBP-associated factor (PCAF), a histone acetyltransferase which reversibly acetylates p53, hence an activator of wtp53. They also observed that PCAF expression was almost absent (10fold less than in control) in 10 out of 15 HMCLs, whereas the remaining 5 cell lines displayed very low expression. In an interesting approach, they transfected MM.1S cells (wtp53) with anti-miR-181s, anti-miR-106b, anti-miR-25, and anti-miR-32, exposed the cells to UV radiation, and measured the expression of p53 and PCAF by q-RT-PCR. After UV treatment, PCAF was accumulated and p53 mRNA expression was increased sixfold after nucleoporation with all antagomiRs simultaneously. Taken together, these findings show that the miR-106b-25 cluster, miR-32, and miR-181a and b target PCAF and through this gene, indirectly control p53 activity and apoptosis in MM cells.

It is important to note that the p53-miRNA axis may also be modulated by exogenous agents which can regulate $\mathrm{p} 53-$ targeting miRNAs. For instance, Gordon et al. [55] reported that some environmental carcinogens may contribute to MM oncogenesis by upregulating p53-targeting miRNAs (oncomiRs); however, this notion requires further support.

\section{Interaction of miRNAs with mtp53: how does miRNA-p53 axis work in the advanced stages of MM?}

Most of the mutations within the TP53 gene are missense giving rise to a full-length $\mathrm{mtp} 53$. This is a unique feature because most other tumor suppressor genes are frequently inactivated by frame shift or nonsense mutations leading to either production of truncated proteins or complete 
elimination of the corresponding gene products [56]. In MM, dysregulation of p53/p53 pathway cannot be due to just TP53 mutations as they occur quite rarely at diagnosis, while wtp53 is frequently dysregulated leading to an imbalance in p53 activity which could be generated through post-transcriptional or post-translational mechanisms. Additionally, our major understanding of the miRNA-p53 interaction in MM pathogenesis is derived from the wtp53, i.e., from studies on MM patients at diagnosis or HMCL harboring wtp53. Thus, it seems conceivable that non-mutational dysfunction of wtp53 bears greater association with $\mathrm{MM}$ pathogenesis. However, the fact that TP53 mutations will become more frequent as MM disease progresses cannot be ignored, with their prevalence reaching $25-30 \%$ in plasma cell leukemia (PCL) $[57,58]$ and $80 \%$ in HMCLs [59]. Therefore, it is crucial to understand whether mtp53-miRNAs interaction also plays any role in MM pathogenesis especially in the advanced stages of the disease. This concept will also help us to find out if such aberrant axis underlies drug resistance in MM patients who are refractory to treatment or at relapse.

In all studies in MM specifically addressing p53-miRNA interaction, functional role of mtp53 is rather elusive or lacking. TP53 mutations have been detected in all HMCLs with low miR-34a and especially del17p.13 in KMS11 cells with undetectable miR-34a; however, miR-34a mimics sensitized them to BTZ raising the probability that miR-34 may function in a p53-independent as well, as indicated elsewhere [29]. On the other hand, reintroducing miR192, miR-194, and miR-215 into wtp53 HMCLs (MM.1S, H929, KMS28BM) led to significant upregulation of CDKN1A and suppression of colony formation but not in mtp53 ones (RPMI-8226 and U266) [60]. These findings provide evidence that miR-192, miR-194, and miR-215 function in a manner dependent upon p53 status, hence, most probably in the presence of a functional wtp53. However, it is still not clear whether resistance of mtp53 HMCLs to these miRNAs is due to compromised miRNA-3'UTR interaction, due to mutant 3'UTR sequence of mtp53, or somehow mtp53 interferes with endogenous or introduced miRNA processing. Also, no clues as to whether mtp53 interaction contributes to drug resistance in MM cells.

To address above issues, specific experimental approaches need to be taken: (1) Generating various TP53 $3^{M U T}$ constructs, introducing them into HMCLs with very low or undetectable p53 to make stable expression, followed by analysis of miRNA profiling with or without anti-myeloma drugs. In support of this idea, transfection of HCT116 colon cancer cell line with tumor-derived p53 mutants decreased mature and precursor miRNA levels of miR-16-1, miR-143, and miR205 [61]. Furthermore, introducing mutant p53R175H into non-small-cell lung cancer cells upregulated miR-128 which in turn sensitized the cells to chemotherapy by suppressing p21 (CDKN1A) [62]. (2) Isolation of primary MM tumor cells from patients at relapse or refractory to treatments for analysis of miRNA expression profiles in the context of their TP53 status and comparison with samples at diagnosis. (3) Analysis of TP53 status and its functional features along with miRNA profiling and 3'UTR binding assays in HMCLs with established drug resistance phenotype such as RPMI-8226R5, MM.1R and comparison with their parental lines. The above approaches will help us to understand if mtp53 in MM cells interacts with miRNAs and whether this interaction modulates drug response of the cells.

Of important note, besides regulating the expression of miRNA genes, wtp53 can affect the maturation (processing) of miRNAs as well. It has been shown that wtp53 directly or indirectly (through p68) interacts with DROSHA complex to promote the processing of specific primary transcript miRNAs (pri-miRNAs) to precursor miRNAs (pre-miRNAs) to increase the levels of the mature miRNAs, e.g., miR-16-1 (which is reported to be a TS-miR in MM [63]), but mtp53 suppressed this interaction [61]. Furthermore, p53 can also interact with DICER1 as evidenced by induced expression of p53 and premature senescence in DICER1 deficiency (incomplete miRNA maturation) [64], whereas mtp53 suppresses DICER1 by inhibiting p63 (a member of p53 family) [65]. Indeed, in mice p63 has been shown to regulate the transcription of Dicer [65]; thus, it would be interesting to investigate whether a global decrease in miRNAs occurs in mtp53 expressing MM cells. Surprisingly, low expression of DICER has been associated with shorter survival and poor prognosis in MM [66] and CLL [67]. Moreover, inactivation of p53 was insufficient to allow untransformed B cells and B cell lymphomas to survive without Dicer [68], providing further support that at least in B cell malignancies interaction of p53 with DICER plays critical role in cell survival and drug response.

Taken all together, mtp53 in contrast to the wtp53 adopts an oncogenic function by interfering with miRNA expression and maturation, another mechanism which might underlie resistance to p53-mediated apoptosis in a mtp53-miRNA network in tumor cells. However, whether this phenomenon explicitly happens in MM cells, to explain the resistance to therapy in MM patients carrying mtp53 demands specific research.

\section{p53-miRNA-epigenetics: a trio governing MM cell viability}

It has been reported that almost all cases of $17 \mathrm{p} 13$ deletion in $\mathrm{MM}$ are mono-allelic and the remaining allele is rarely mutated [69]. Whether the remaining allele is inactivated by other mechanisms like DNA methylation or MDM2 upregulation leading to suppressed p53 pathway in MM was explored by Teoh et al. [69]. They demonstrated that p53 
haplo-insufficiency accounted for the compromised response of MM cells to genotoxic stress when one allele of TP53 is lost. They treated a panel of HMCLs with different p53 status with the genotoxic agent etoposide and observed that wt/wt cells (H929) are more sensitive to drug-induced apoptosis than wt/- cells (XG6), mtp53 cells (RPMI-8226 and U266) showed negligible cell death, and cells with very low or completely absent p53 (JJN3 and KMS18) were resistant to drug-induced apoptosis. The authors also identified that hypermethylation of p53 promoter is the cause of inactivation of the remaining TP53 allele in wt/- MM cells. Promoter hypermethylation has also been reported for some p53-induced miRNAs in MM cells, such as miR-34a, miR$192,194,215$, which in fact have tumor suppressor activity $[28,38]$. While it is conceivable that p53 suppression in MM cells may be partly due to overexpression of some oncomiRs, it remains to be elucidated whether oncomiRs contribute to p53 suppression by triggering promoter hypermethylation in MM. Epigenetic mechanisms may work indirectly on miRNAs as well to affect p53 activity/abundance. It is reported that some oncomiRs like miR-106b 25 cluster and miR-125b which negatively regulate TP53 gene (see above) are reduced by EZH2 inhibition. This was indicated by the observation that EZH2 indirectly upregulated those miRNAs by suppressing their transactivators [70]. Pichiorri et al. also describe that miR-192, 194, and 215 are epigenetically silenced in MM cells by promoter hypermethylation. Downregulation of these miRNAs leads to increased MDM2 mRNA and protein expression and decreased p53 level. Interestingly, epigenetic silencing of miR-137 in MM cells lead to MDM2-mediated degradation of p53 through upregulation of AURKA which was found to be a direct target of miR-137. Introducing miR-137 mimics into drug-resistant MM cells could re-sensitize them to anti-myeloma drugs by inhibition of p53 degradation and inducing apoptosis [71]. Therefore, it seems conceivable that a combination of epigenetic silencing of p53-activated miRNAs and p53 in the context of myeloma tumor further contributes to dysregulation of p53 pathway and enforced survival of MM cells.

\section{The effect of bone marrow stroma on p53-miRNA interaction: implication for mechanism of cell adhesion- mediated drug resistance (CAMDR)}

It is reported that tumor microenvironment may also contribute to non-mutational dysregulation of wtp53 in tumor cells. A few studies have shown that bone marrow stromal cells (BMSCs) can modulate p53-related miRNAs and negatively affect p53 function and expression in MM cells through direct or indirect cell-cell interaction [72, 73]. This phenomenon may be partly supported by the fact that IL-6, the main survival factor for MM cells secreted by BMSCs, indeed suppresses p53 function [74], and integrin activation following tumor cell-stroma adhesion modulates p53 function [75]. Moreover, it is established that integrin engagement following cell-cell adhesion drives cell-cycle machinery [76]. Hence, modulation of p53-targeting miRNAs by BMSCs in MM seems plausible, as they are established as integral part of p53 regulatory network (the stroma concept is illustrated in Fig. 2).

Notably, the few studies performed to date in MM addressing specifically the effect of BMSCs on expression and function of p53 used only wtp53 MM cells. The most probable reason is to understand whether stroma regulates/modulates p53 "with a normal function" in the context of drugs, as it is reported that at advanced stages of the disease (where the rate of p53 mutation will also increase) MM cells may become stroma-independent/unresponsive [77]. Leotta et al. [21] showed that adhesion of wtp53 MM cells to BMSCs strongly upregulated miR-125a-5p level, while reduced p53 expression; however, whether interaction between these two contributes to drug resistance in stroma context was not pursued. Furthermore, in a preliminary work, we showed that when wtp53 harboring MM.1S cells adhered to BMSCs, the mRNA of TP53, BAX, NOXA, MDM2, and PTEN was downregulated, while p53-targeting miRNAs, miR-125a, miR-125b, miR-25, and miR-181b were upregulated. On the other hand, some p53-regulated miRNAs such as miR-15a and miR-16 were downregulated. Above modulations happened in the context of BTZ which gives an implication of involvement of p53/miRNA interaction in stroma-induced drug resistance in MM.

Interestingly, recent studies have identified an important role for exosomes secreted from BMSCs in modulation of wtp53 function and in contribution to stroma-mediated drug resistance in MM [78]. These extra cellular vesicles indeed contain mRNAs and miRNAs which through unclarified mechanisms affect various signaling pathways including p53 in MM cells. Strikingly, p53 has been reported to play a key role in exosome secretion [79]. Furthermore, p53 status of stromal cells in tumor environment is reported to influence the drug response of tumor cells [80]; thus, it would be interesting to explore if p53 status in BMSCs from MM patients affects the level of protection for MM cells or their response to anti-myeloma drugs.

Taken together, BMSCs have suppressive effects on wtp53 expression and function probably by modulating p53-related miRNAs. It remains to be elucidated how BMSCs generate such effects; however, induction of an epigenetic silencing mechanism in $\mathrm{MM}$ cells may be speculated. A study conducted by Amodio et al. [81] shows that BMSCs upregulate DNMT3A and DNMT3A (enzymes responsible for DNA methylation) in MM cells following direct adhesion implying that BMSCs may trigger a global DNA methylation in MM cells; however, this requires further exploration. 


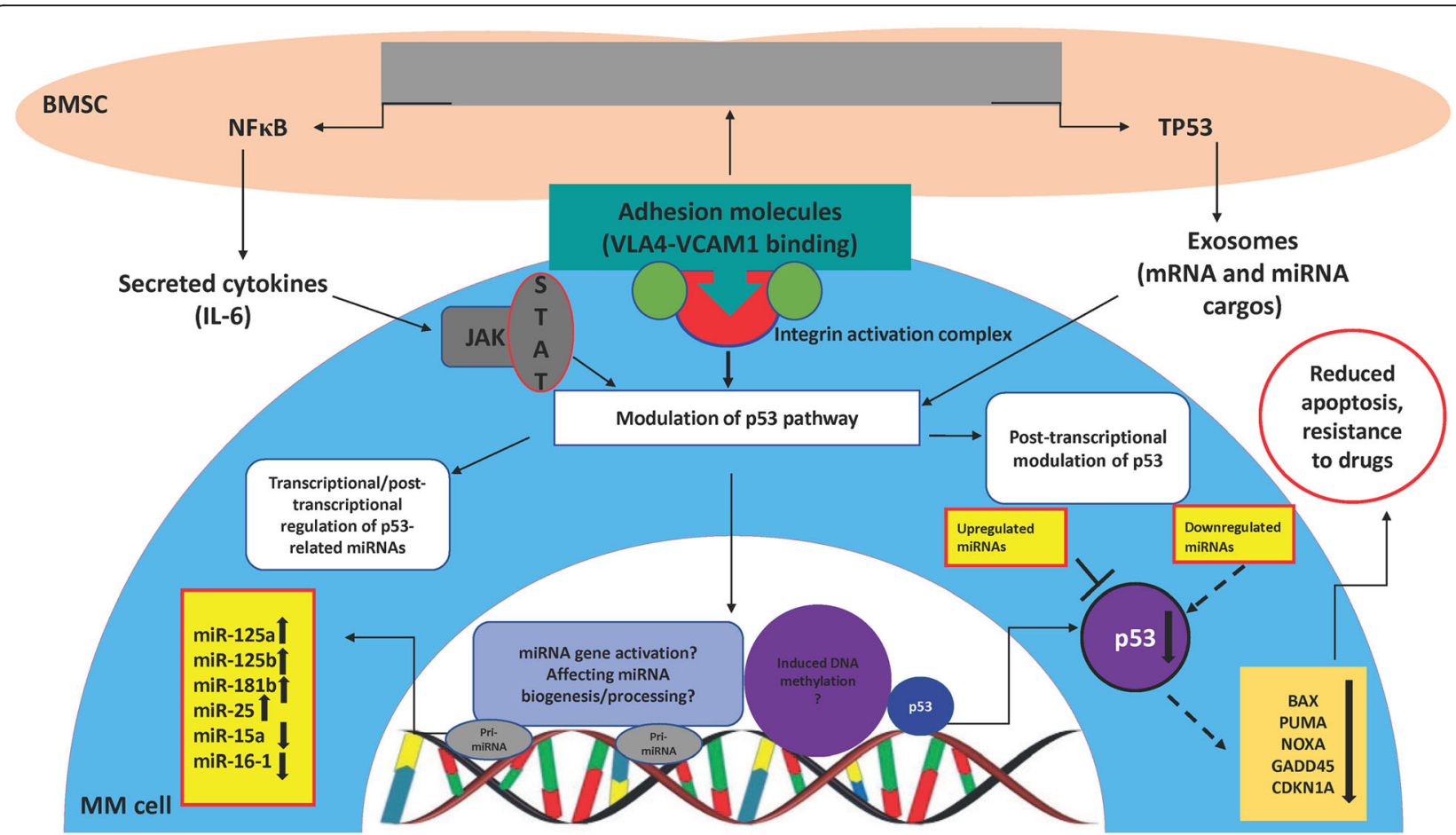

Fig. 2 Proposed scheme for the role of BMSCs in modulation of p53-miRNA interaction in association with stroma-induced drug resistance in MM. Following interaction between BMSCs and MM cells, p53 mRNA and protein and some p53-related TS-miRs (miR-15a/16-1) will be downregulated. On the other hand, the expression of some oncomiRs which negatively regulate p53 such as miR-125a, b will be promoted. Interestingly, exosomes released from BMSCs also contain miRNAs which affect p53 pathway. The outcome can be attenuation of p53-mediated apoptotic effects and drug resistance (CAMDR). It is established that integrin activation and cytokine signaling pathways will cross-talk with p53 pathway which ends in modulation of the pathway and also post-transcriptional changes in p53. However, it requires thorough investigations whether pathways activated following BMSC-MM cell interaction somehow impinge on transcriptional modulation of TP53 or miRNA genes in the nucleus through epigenetic mechanisms, miRNA biogenesis, or gene activation

\section{miRNA targeting of p53 signaling pathway: therapeutic applications}

Since p53 is in the middle of a highly complex and coordinated network involved in challenging any oncogenic input which may jeopardize genome integrity, reactivation of p53 or maintaining its activity seems to be a promising strategy to treat MM. However, this concept bears significance for MM patients carrying wild type but dysfunctional p53, as mtp53 generates a higher propensity for chemoresistance [82]. Consequently, developing powerful strategies to overcome such complication requires exploring the function of $\mathrm{mt} 53$ in MM cells. The approaches applied to targeting strategies for the p53 pathway in MM using small molecule compounds (which reportedly activate p53 or restore wild-type activity of mtp53) have been reviewed before [6]. Additionally, limitations concerning such strategies have been put forward as well [83]. Some caveats depending on type and function of the small molecule include DNA damage due to DNA intercalation, independency on p53 of their lethality (or killing effects may not be clearly through p53), inability to trigger post-translational modifications necessary to obtain a p53 response, and finally the sum of all these methodologies does not cover all TP53 alleles.
Pre-clinical evidences obtained from administration of miRNA mimics (miR-34a [27]) and miR-192, miR-194, 215 [60]), provide promising platforms to support the concept of applying p53-activating miRNAs to MM therapeutic strategies. This strategy will be especially helpful when p53-activating miRNAs are combined with small molecules like nutlin-3a or MI-219 which increase the level of p53 by inhibiting MDM2 [60]. AntagomiRs against miR-125a or miR-125a have not been explored in $\mathrm{MM}$ xenograft models yet, but the in vitro observations related to them indicate their potential for translation into in vivo studies. On the other hand, on top of all are still the issues related to application of miRNAs to the clinic [84]. The major concerns are related to delivery strategies to avoid off-target effects, reduced miRNA activity, and production of toxic metabolites. This area demands continuous research to make these small molecules clinically applicable.

\section{Concluding remarks and future perspectives}

In all hematologic malignancies including MM, p53 abnormalities and dysregulation are perhaps the most extensively studied among other critical tumor suppressors 
or oncogenes. A variety of small molecule compounds with the ability to restore p53 activity have been applied alone or combined with anti-myeloma drugs to MM pre-clinical models yielding promising results $[7,8,85$, 86]. However, MM patients are still dealing with therapy resistance or relapse, which is the most challenging aspect of MM pathogenesis. To that end, contribution of dysfunctional p53 or p53 pathway to MM drug resistance has been insufficiently investigated and demands more detailed research to understand the mechanisms underlying dysregulation of p53 in MM patients associated with disease progression and drug resistance.

Current findings on substantial contribution of miRNAs to tumor suppressor activity of p53 in cancers including $\mathrm{MM}$ provide convincing evidence that miRNAs are now part of the p53 regulatory network. That being said, more thorough investigations are still required to establish miRNAs as important regulatory hubs in p53 network to help decipher the mechanism underlying therapy resistance related to dysregulated p53 in MM. Importantly, it is still unknown whether miRNAs have a crucial role in mtp53 function explicitly in $\mathrm{MM}$ and whether their effects are dependent on p53 status.

\section{Conclusion}

In conclusion, miRNAs play a key role in p53 network and in the regulation of p53 tumor suppressor activity. Given further comprehensive research, interaction of miRNAs with wtp53 or mtp53 will shed light on the mechanisms underlying drug resistance and relapse in MM. This will indeed provide novel venues for therapeutic targeting of dysregulated p53 pathway in MM and particularly for overcoming drug resistance due to a dysfunctional p53.

\footnotetext{
Abbreviations

3'UTR: 3' untranslated region; ALL: Acute lymphoblastic leukemia; AML: Acute myelogenous leukemia; BCL-2: B cell lymphoma-2; BMF: BCl-2-modifying factor; BMSCs: Bone marrow stromal cells; BTZ: Bortezomib; CDK4 and CDK6: Cyclin-dependent kinases 4 and 6; CDKN1A: Cyclin-dependent kinase 1 A; CLL: Chronic lymphocytic leukemia; DEX: Dexamethasone; DLBCL: Diffuse large B cell lymphoma; DNMT3A and DNMT3A: DNA (cytosine-5-)-methyltransferase $3 \mathrm{~A}$ and B; FISH: Fluorescent in situ hybridization; GADD45: Growth arrest and DNA damage 45; HMCLs: Human myeloma cell lines; KLF13: Kruppel-like factor 13; MDM2: Mouse double minute 2 homolog; MM: Multiple myeloma; mt: Mutant; PCAF: p300-CBPassociated factor; PSMD10: 265 proteasome non-ATPase regulatory subunit 10; PTEN: Phosphatase and tensin homolog; PUMA: p53-upregulated modulator of apoptosis; RISC: RNA-induced silencing complex; SIRT1: NAD-dependent deacetylase sirtuin-1; TSmiR: Tumor suppressor miRNA; wt: Wild type; YY1: Ying Yang 1
}

\section{Acknowledgements}

Not applicable

\section{Funding}

The study was supported in part by the grants from Leukemia and Lymphoma Research Society of Canada (LLSC), Cancer Research Society (CRS). The funding agencies played no role in the design, reviewing, or writing the manuscript.
Availability of data and materials

Please contact the author for data requests.

\section{Authors' contributions}

JA and NR drafted the manuscript. LL and WC participated in the design of the study. HC conceived of the study and participated in its design and coordination and helped to draft the manuscript. All authors read and approved the final manuscript.

Ethics approval and consent to participate

Not applicable

Consent for publication

NA

Competing interests

The authors declare that they have no competing interests.

\section{Publisher's Note}

Springer Nature remains neutral with regard to jurisdictional claims in published maps and institutional affiliations.

\section{Author details}

${ }^{1}$ Division of Molecular and Cellular Biology, Toronto General Research Institute, Toronto, Canada. ${ }^{2}$ Department of Laboratory Medicine \& Pathobiology, University of Toronto, Toronto, Canada. ${ }^{3}$ Department of Hematology, Beijing Chaoyang Hospital, Capital Medical University, Beijing, China. ${ }^{4}$ Department of Laboratory Hematology and Medical Oncology, University Health Network, 200 Elizabeth Street, 11E-413, Toronto, ON M5G 2C4, Canada.

Received: 25 September 2017 Accepted: 18 October 2017

Published online: 26 October 2017

\section{References}

1. Tessoulin B, Eveillard M, Lok A, Chiron D, Moreau P, Amiot M, et al. p53 dysregulation in B-cell malignancies: more than a single gene in the pathway to hell. Blood Rev. 2017;04

2. Lionetti M, Barbieri M, Manzoni M, Fabris S, Bandini C, Todoerti K, et al. Molecular spectrum of TP53 mutations in plasma cell dyscrasias by next generation sequencing: an Italian cohort study and overview of the literature. Oncotarget. 2016;7(16):21353-61.

3. Chen MH, Qi CX, Saha MN, Chang H. p53 nuclear expression correlates with hemizygous TP53 deletion and predicts an adverse outcome for patients with relapsed/refractory multiple myeloma treated with lenalidomide. Am J Clin Pathol. 2012;137(2):208-12.

4. Chang H, Qi C, Yi QL, Reece D, Stewart AK. p53 gene deletion detected by fluorescence in situ hybridization is an adverse prognostic factor for patients with multiple myeloma following autologous stem cell transplantation. Blood. 2005;105(1):358-60.

5. Chang H, Yeung J, Qi C, Xu W. Aberrant nuclear p53 protein expression detected by immunohistochemistry is associated with hemizygous P53 deletion and poor survival for multiple myeloma. Br J Haematol. 2007; 138(3):324-9.

6. Saha MN, Micallef J, Qiu L, Chang H. Pharmacological activation of the p53 pathway in haematological malignancies. J Clin Pathol. 2010;63(3):204-9.

7. Saha MN, Jiang H, Jayakar J, Reece D, Branch DR, Chang H. MDM2 antagonist nutlin plus proteasome inhibitor velcade combination displays a synergistic anti-myeloma activity. Cancer Biol Ther. 2010;9(11):936-44.

8. Saha MN, Jiang H, Mukai A, Chang H. RITA inhibits multiple myeloma cell growth through induction of p53-mediated caspase-dependent apoptosis and synergistically enhances nutlin-induced cytotoxic responses. Mol Cancer Ther. 2010;9(11):3041-51.

9. Nguyen D, Liao W, Zeng SX, Lu H. Reviving the guardian of the genome: small molecule activators of p53. Pharmacol Ther. 2017;27

10. Stuhmer T, Chatterjee M, Hildebrandt M, Herrmann P, Gollasch H, Gerecke C, et al. Nongenotoxic activation of the p53 pathway as a therapeutic strategy for multiple myeloma. Blood. 2005;106(10):3609-17.

11. He L, Hannon GJ. MicroRNAs: small RNAs with a big role in gene regulation. Nat Rev Genet. 2004;5(7):522-31. 
12. Friedman RC, Farh KK, Burge CB, Bartel DP. Most mammalian mRNAs are conserved targets of microRNAs. Genome Res. 2009;19(1):92-105.

13. Jones M, Lal A. MicroRNAs, wild-type and mutant p53. RNA Biol. 2012;9(6):781-91.

14. Leung AK, Sharp PA. microRNAs: a safeguard against turmoil? Cell. 2007; 130(4):581-5.

15. Leung AK, Sharp PA. MicroRNA functions in stress responses. Mol Cell. 2010; 40(2):205-15.

16. Abdi J, Jian $\mathrm{H}$, Chang $\mathrm{H}$. Role of micro-RNAs in drug resistance of multiple myeloma. Oncotarget. 2016;7(37):60723-35.

17. Abdi J, Qiu L, Chang H. Micro-RNAs, new performers in multiple myeloma bone marrow microenvironment. Biomark Res. 2014;2:10.

18. Rastgoo N, Abdi J, Hou J, Chang H. Role of epigenetics-microRNA axis in drug resistance of multiple myeloma. J Hematol Oncol. 2017;10(1):121.

19. Bi C, Chng WJ. MicroRNA: important player in the pathobiology of multiple myeloma. Biomed Res Int. 2014;2014:521586.

20. Murray MY, Rushworth SA, Zaitseva L, Bowles KM, Macewan DJ. Attenuation of dexamethasone-induced cell death in multiple myeloma is mediated by miR-125b expression. Cell Cycle. 2013;12(13):2144-53.

21. Leotta M, Biamonte L, Raimondi L, Ronchetti D, Di Martino MT, Botta C, et al. A p53-dependent tumor suppressor network is induced by selective miR-125a-5p inhibition in multiple myeloma cells. J Cell Physiol 2014; 229(12): 2106-2116.

22. Deng Q, Becker L, Ma X, Zhong X, Young K, Ramos K, et al. The dichotomy of p53 regulation by noncoding RNAs. J Mol Cell Biol. 2014;6(3):198-205.

23. Feng Z, Zhang C, Wu R, Hu W. Tumor suppressor p53 meets microRNAs. J Mol Cell Biol. 2011;3(1):44-50.

24. Hermeking $\mathrm{H}$. MicroRNAs in the p53 network: micromanagement of tumour suppression. Nat Rev Cancer. 2012;12(9):613-26.

25. Navarro F, Lieberman J. miR-34 and p53: new insights into a complex functional relationship. PLoS One. 2015;10(7):e0132767.

26. Rokavec M, Li H, Jiang L, Hermeking $H$. The p53/miR-34 axis in development and disease. J Mol Cell Biol. 2014;6(3):214-30.

27. Di Martino MT, Leone E, Amodio N, Foresta U, Lionetti M, Pitari MR, et al. Synthetic miR-34a mimics as a novel therapeutic agent for multiple myeloma: in vitro and in vivo evidence. Clin Cancer Res. 2012;18(22):6260-70.

28. Wong KY, Huang $X$, Chim CS. DNA methylation of microRNA genes in multiple myeloma. Carcinogenesis. 2012;33(9):1629-38.

29. Stebner ENP, Johnson J, Gratton KJ, Ren L, Duggan P, Stewart DA, Bahlis N. Mir-34a sensitizes multiple myeloma (MM) cells to the proteasome inhibitor bortezomib. Blood. 2011;118(21):138-38.

30. Okada N, Lin CP, Ribeiro MC, Biton A, Lai G, He X, et al. A positive feedback between p53 and miR-34 miRNAs mediates tumor suppression. Genes Dev. 2014;28(5):438-50.

31. Zenz T, Mohr J, Eldering E, Kater AP, Buhler A, Kienle D, et al. miR-34a as part of the resistance network in chronic lymphocytic leukemia. Blood. 2009;113(16):3801-8.

32. Misiewicz-Krzeminska I, Sarasquete ME, Quwaider D, Krzeminski P, Ticona FV, Paino T, et al. Restoration of microRNA-214 expression reduces growth of myeloma cells through positive regulation of P53 and inhibition of DNA replication. Haematologica. 2013;98(4):640-8.

33. Hao M, Zang M, Zhao L, Deng S, Xu Y, Qi F, et al. Serum high expression of miR-214 and miR-135b as novel predictor for myeloma bone disease development and prognosis. Oncotarget. 2016;7(15):19589-600.

34. Xu CX, Xu M, Tan L, Yang H, Permuth-Wey J, Kruk PA, et al. MicroRNA miR214 regulates ovarian cancer cell stemness by targeting p53/Nanog. J Biol Chem. 2012;287(42):34970-8.

35. Chiang Y, Song Y, Wang Z, Liu Z, Gao P, Liang J, et al. microRNA-192, -194 and -215 are frequently downregulated in colorectal cancer. Exp Ther Med. 2012;3(3):560-6.

36. Khella HW, Bakhet M, Allo G, Jewett MA, Girgis AH, Latif A, et al. miR-192, miR-194 and miR-215: a convergent microRNA network suppressing tumor progression in renal cell carcinoma. Carcinogenesis. 2013;34(10):2231-9.

37. Braun CJ, Zhang X, Savelyeva I, Wolff S, Moll UM, Schepeler T, et al. p53responsive micrornas 192 and 215 are capable of inducing cell cycle arrest. Cancer Res. 2008;68(24):10094-104.

38. Pichiorri F, Suh SS, Rocci A, De Luca L, Taccioli C, Santhanam R, et al. Downregulation of p53-inducible microRNAs 192, 194, and 215 impairs the p53/MDM2 autoregulatory loop in multiple myeloma development. Cancer Cell 2016;30(2):349-351.
39. Kumar M, Lu Z, Takwi AA, Chen W, Callander NS, Ramos KS, et al. Negative regulation of the tumor suppressor p53 gene by microRNAs. Oncogene. 2011;30(7):843-53

40. Le MT, Teh C, Shyh-Chang N, Xie H, Zhou B, Korzh V, et al. MicroRNA-125b is a novel negative regulator of p53. Genes Dev. 2009;23(7):862-76.

41. Hu W, Chan CS, Wu R, Zhang C, Sun Y, Song JS, et al. Negative regulation of tumor suppressor p53 by microRNA miR-504. Mol Cell. 2010;38(5):689-99.

42. Swarbrick A, Woods SL, Shaw A, Balakrishnan A, Phua Y, Nguyen A, et al. miR-380-5p represses p53 to control cellular survival and is associated with poor outcome in MYCN-amplified neuroblastoma. Nat Med. 2010;16(10):1134-40.

43. Neveu P, Kye MJ, Qi S, Buchholz DE, Clegg DO, Sahin M, et al. MicroRNA profiling reveals two distinct p53-related human pluripotent stem cell states. Cell Stem Cell. 2010;7(6):671-81.

44. Shaham L, Binder V, Gefen N, Borkhardt A, Izraeli S. MiR-125 in normal and malignant hematopoiesis. Leukemia. 2012;26(9):2011-8.

45. Shi XB, Xue L, Ma AH, Tepper CG, Kung HJ, White RW. miR-125b promotes growth of prostate cancer xenograft tumor through targeting pro-apoptotic genes. Prostate. 2011;71(5):538-49.

46. Zhou M, Liu Z, Zhao Y, Ding Y, Liu H, Xi Y, et al. MicroRNA-125b confers the resistance of breast cancer cells to paclitaxel through suppression of proapoptotic Bcl-2 antagonist killer 1 (Bak1) expression. J Biol Chem. 2010; 285(28):21496-507.

47. O'Connell RM, Chaudhuri AA, Rao DS, Gibson WS, Balazs AB, Baltimore D. MicroRNAs enriched in hematopoietic stem cells differentially regulate longterm hematopoietic output. Proc Natl Acad Sci U S A. 2010;107(32):14235-40.

48. Bousquet M, Harris MH, Zhou B, Lodish HF. MicroRNA miR-125b causes leukemia. Proc Natl Acad Sci U S A. 2010;107(50):21558-63.

49. Akbari Moqadam F, Lange-Turenhout EA, Aries IM, Pieters R, den Boer ML. MiR-125b, miR-100 and miR-99a co-regulate vincristine resistance in childhood acute lymphoblastic leukemia. Leuk Res. 2013;37(10):1315-21.

50. Zhang H, Luo XQ, Feng DD, Zhang XJ, Wu J, Zheng YS, et al. Upregulation of microRNA-125b contributes to leukemogenesis and increases drug resistance in pediatric acute promyelocytic leukemia. Mol Cancer. 2011;10:108.

51. Banzhaf-Strathmann J, Edbauer D. Good guy or bad guy: the opposing roles of microRNA 125b in cancer. Cell Commun Signal. 2014;12:30.

52. Troppan K, Wenzl K, Deutsch A, Ling H, Neumeister P, Pichler M. MicroRNAs in diffuse large B-cell lymphoma: implications for pathogenesis, diagnosis, prognosis and therapy. Anticancer Res. 2014;34(2):557-64.

53. Bai H, Zhou L, Wang C, Xu X, Jiang J, Qin Y, et al. Involvement of miR-125a in resistance to daunorubicin by inhibiting apoptosis in leukemia cell lines. Tumour Biol. 2017;39(4):1010428317695964.

54. Pichiorri F, Suh SS, Ladetto M, Kuehl M, Palumbo T, Drandi D, et al. MicroRNAs regulate critical genes associated with multiple myeloma pathogenesis. Proc Natl Acad Sci U S A. 2008;105(35):12885-90.

55. Gordon MW, Yan F, Zhong X, Mazumder PB, Xu-Monette ZY, Zou D, et al. Regulation of p53-targeting microRNAs by polycyclic aromatic hydrocarbons: implications in the etiology of multiple myeloma. Mol Carcinog. 2015;54(10):1060-9.

56. Weisz L, Oren M, Rotter V. Transcription regulation by mutant p53. Oncogene. 2007;26(15):2202-11.

57. Cifola I, Lionetti M, Pinatel E, Todoerti K, Mangano E, Pietrelli A, et al. Wholeexome sequencing of primary plasma cell leukemia discloses heterogeneous mutational patterns. Oncotarget. 2015;6(19):17543-58.

58. Tiedemann RE, Gonzalez-Paz N, Kyle RA, Santana-Davila R, Price-Troska T, Van Wier SA, et al. Genetic aberrations and survival in plasma cell leukemia. Leukemia 2008; 22(5): 1044-1052.

59. Mazars GR, Portier M, Zhang XG, Jourdan M, Bataille R, Theillet C, et al. Mutations of the p53 gene in human myeloma cell lines. Oncogene. 1992;7(5):1015-8

60. Pichiorri F, Suh SS, Rocci A, De Luca L, Taccioli C, Santhanam R, et al. Downregulation of p53-inducible microRNAs 192, 194, and 215 impairs the p53/MDM2 autoregulatory loop in multiple myeloma development. Cancer Cell 2010; 18(4): 367-381.

61. Suzuki HI, Yamagata K, Sugimoto K, Iwamoto T, Kato S, Miyazono K. Modulation of microRNA processing by p53. Nature. 2009;460(7254):529-33.

62. Donzelli S, Fontemaggi G, Fazi F, Di Agostino S, Padula F, Biagioni F, et al. MicroRNA-128-2 targets the transcriptional repressor E2F5 enhancing mutant p53 gain of function. Cell Death Differ. 2012;19(6):1038-48.

63. Li F, Xu Y, Deng S, Li Z, Zou D, Yi S, et al. MicroRNA-15a/16-1 cluster located at chromosome $13 q 14$ is down-regulated but displays different expression 
pattern and prognostic significance in multiple myeloma. Oncotarget. 2015; 6(35):38270-82.

64. Mudhasani R, Zhu Z, Hutvagner G, Eischen CM, Lyle S, Hall LL, et al. Loss of miRNA biogenesis induces p19Arf-p53 signaling and senescence in primary cells. J Cell Biol. 2008;181(7):1055-63.

65. Su X, Chakravarti D, Cho MS, Liu L, Gi YJ, Lin YL, et al. TAp63 suppresses metastasis through coordinate regulation of Dicer and miRNAs. Nature. 2010;467(7318):986-90.

66. Sarasquete ME, Gutierrez NC, Misiewicz-Krzeminska I, Paiva B, Chillon MC Alcoceba $\mathrm{M}$, et al. Upregulation of Dicer is more frequent in monoclonal gammopathies of undetermined significance than in multiple myeloma patients and is associated with longer survival in symptomatic myeloma patients. Haematologica. 2011;96(3):468-71.

67. Zhu DX, Fan L, Lu RN, Fang C, Shen WY, Zou ZJ, et al. Downregulated Dicer expression predicts poor prognosis in chronic lymphocytic leukemia. Cancer Sci. 2012;103(5):875-81.

68. Adams CM, Eischen CM. Inactivation of $\mathrm{p} 53$ is insufficient to allow B cells and B-cell lymphomas to survive without Dicer. Cancer Res. 2014;74(14):3923-34.

69. Teoh PJ, Chung TH, Sebastian S, Choo SN, Yan J, Ng SB, et al. p53 haploinsufficiency and functional abnormalities in multiple myeloma. Leukemia. 2014;28(10):2066-74.

70. Alzrigat M, Parraga AA, Agarwal P, Zureigat H, Osterborg A, Nahi H, et al. EZH2 inhibition in multiple myeloma downregulates myeloma associated oncogenes and upregulates microRNAs with potential tumor suppressor functions. Oncotarget. 2017;8(6):10213-24.

71. Qin Y, Zhang S, Deng S, An G, Qin X, Li F, et al. Epigenetic silencing of miR137 induces drug resistance and chromosomal instability by targeting AURKA in multiple myeloma. Leukemia. 2017;31(5):1123-35.

72. Ooi MG, Hayden PJ, Kotoula V, McMillin DW, Charalambous E, Daskalaki E, et al. Interactions of the $\mathrm{Hdm} 2 / \mathrm{p} 53$ and proteasome pathways may enhance the antitumor activity of bortezomib. Clin Cancer Res. 2009;15(23):7153-60.

73. McMillin DW, Delmore J, Weisberg E, Negri JM, Geer DC, Klippel S, et al. Tumor cell-specific bioluminescence platform to identify stroma-induced changes to anticancer drug activity. Nat Med. 2010;16(4):483-9.

74. Hodge DR, Peng B, Cherry JC, Hurt EM, Fox SD, Kelley JA, et al. Interleukin 6 supports the maintenance of p53 tumor suppressor gene promoter methylation. Cancer Res. 2005:65(11):4673-82.

75. Lewis JM, Truong TN, Schwartz MA. Integrins regulate the apoptotic response to DNA damage through modulation of p53. Proc Natl Acad Sci U S A. 2002; 99(6):3627-32

76. Moreno-Layseca P, Streuli CH. Signalling pathways linking integrins with cell cycle progression. Matrix Biol. 2014;34:144-53.

77. Kuehl WM, Bergsagel PL. Molecular pathogenesis of multiple myeloma and its premalignant precursor. J Clin Invest. 2012;122(10):3456-63.

78. Wang J, Hendrix A, Hernot S, Lemaire M, De Bruyne E, Van Valckenborgh E, et al. Bone marrow stromal cell-derived exosomes as communicators in drug resistance in multiple myeloma cells. Blood 2014; 124(4): 555-566.

79. Yu X, Harris SL, Levine AJ. The regulation of exosome secretion: a novel function of the p53 protein. Cancer Res. 2006;66(9):4795-801.

80. Hasebe T, Tamura N, Okada N, Hojo T, Akashi-Tanaka S, Shimizu C, et al. p53 expression in tumor-stromal fibroblasts is closely associated with the nodal metastasis and outcome of patients with invasive ductal carcinoma who received neoadjuvant therapy. Hum Pathol. 2010;41 (2):262-70.

81. Amodio N, Leotta M, Bellizzi D, Di Martino MT, D'Aquila P, Lionetti M, et al. DNA-demethylating and anti-tumor activity of synthetic miR-29b mimics in multiple myeloma. Oncotarget 2012; 3(10): 1246-1258.

82. Chng WJ, Price-Troska T, Gonzalez-Paz N, Van Wier S, Jacobus S, Blood E, et al. Clinical significance of TP53 mutation in myeloma. Leukemia 2007; 21(3): 582-584.

83. Yu X, Narayanan S, Vazquez A, Carpizo DR. Small molecule compounds targeting the p53 pathway: are we finally making progress? Apoptosis. 2014;19(7):1055-68.

84. Rupaimoole R, Han HD, Lopez-Berestein G, Sood AK. MicroRNA therapeutics: principles, expectations, and challenges. Chin J Cancer. 2011;30(6):368-70.

85. Saha MN, Jiang H, Yang Y, Reece D, Chang H. PRIMA-1Met/APR-246 displays high antitumor activity in multiple myeloma by induction of p73 and Noxa. Mol Cancer Ther. 2013;12(11):2331-41.

86. Tessoulin B, Descamps G, Moreau P, Maiga S, Lode L, Godon C, et al. PRIMA1 Met induces myeloma cell death independent of p53 by impairing the GSH/ROS balance. Blood. 2014;124(10):1626-36.

\section{Submit your next manuscript to BioMed Central and we will help you at every step:}

- We accept pre-submission inquiries

- Our selector tool helps you to find the most relevant journal

- We provide round the clock customer support

- Convenient online submission

- Thorough peer review

- Inclusion in PubMed and all major indexing services

- Maximum visibility for your research

Submit your manuscript at www.biomedcentral.com/submit
Biomed Central 